\title{
Modeling of Rotating Shaft with Partial Rubbing
}

\author{
Tatsuro Ishibashi ${ }^{1}$ Tadao Kawai ${ }^{2}$ \\ ${ }^{1}$ Meidensha Corporation, Japan, ishibashi - tat@mb .meidensha .co.jp \\ ${ }^{2}$ Department of Mechanical \& Physical Engineering, Osaka City University, Japan, \\ kawai@osaka-cu.ac.jp
}

\begin{abstract}
We have created the rotating machinery library to carry out analytical investigations for diagnosis by transfer matrix method in Modelica. In this paper, rubbing components for partial rub are implemented in our rotating machinery library. In this research, the model in which the rotor come into contact from a non-contact state by a pulsating external force is analyzed. The relationship between the contact configurations and the generation of various kinds of vibration is investigated. We validated the rubbing model in one side contact case with a rotor kit. By simulation, we reproduced the time history, the orbit and the full spectrum characteristics of the rotating shaft measured by the experiment precisely.
\end{abstract}

Keywords: Rotor Dynamics, Rubbing, Contact, Friction, Subharmonics

\section{Introduction}

To improve efficiency in rotating machinery, the clearance between rotors and casings has become smaller and smaller. However, it increases risk of rubbing i.e. contact between rotating and stationary elements of a machine. It is mainly resulted from the mass unbalance, turbine or compressor blade failure, defective bearing, or rotor misalignment. The rubimpacting vibration of a rotor system shows a very complicated phenomenon including not only the periodic motion but also the quasi-periodic and chaotic motions. When the rub-impact happens, the partial rub arises at first. During a whole period, the rub and impact interactions occur between rotors and stators (i.e. casings) once or fewer times. Gradual deterioration of the partial rub will lead to the full rub, and then the vibration will affect the normal operation of the machines negatively. Thus, the rubbing phenomenon is one of the main malfunctions in rotating machines and causes the breakdown of machines.

Because of serious damage of rubbing, many researchers have studied this problem from different aspects (Ehrich, 1966; Beatty, 1985; Choi and Noah, 1987). Much attention has been given to the nonlinear dynamics of the rub-impacting rotor system. A contact force of rubbing between a rotor and a casing has been modeled as a piecewise linear spring and damper model.
The relationship between the contact configurations and the generation of various kinds of vibration, such as "collision type synchronous vibration", "sub harmonic vibration", etc. has been studied, both theoretically and experimentally (Watanabe et al, 2004; Watanabe et al, 2005)

We have created the rotating machinery library by transfer matrix method in Modelica (Ishibashi et al, 2017). By transfer matrix method, the rotating shaft is decomposed into rotors, shafts, journals, couplings, housings and supports. The 5 DOF rotor dynamics model components have common faults of rotating machinery systems such as static and dynamic unbalance, shaft bending, and faulty bearing. Basic components are reusable, and their parameters can be simply modified. Even if it is not a Jeffcott (i.e. symmetrical) rotor system, this library makes it easy to analyze dynamics of rotating machinery. The objective of creating this library is to carry out analytical investigations in order to gain some insight into the diagnostics of rotating machinery.

Many papers have been written regarding modeling contact phenomena in the Modelica language. The contact models can be roughly classified into two types, collision of multibody objects and contact of gears. The former is handled in the following papers. A solution based on a collision handling software called Solid was described in (Otter et al, 2005). The paper (Oestersötebier et al, 2014) introduced non-central contact blocks in which the contact surfaces were defined. (Hofmann et al, 2014) discussed the use of the Bullet Physics Library.

The latter is handled in the following papers. One work is (van der Linden, 2012) where the 3 DOF elastic gear contact model was implemented in the Planar Mechanics library. A much more detailed approach was taken by (Kosenko and Gusev, 2011) and further improved in (Kosenko and Gusev, 2012), where the forces between gears were modelled with high detail in a Modelica environment. (Dahl et al, 2017) integrated the gear contact model in the MultiBody library from the Modelica Standard library.

In this paper, rubbing components for partial rub are implemented in our rotating machinery library. Rubbing 

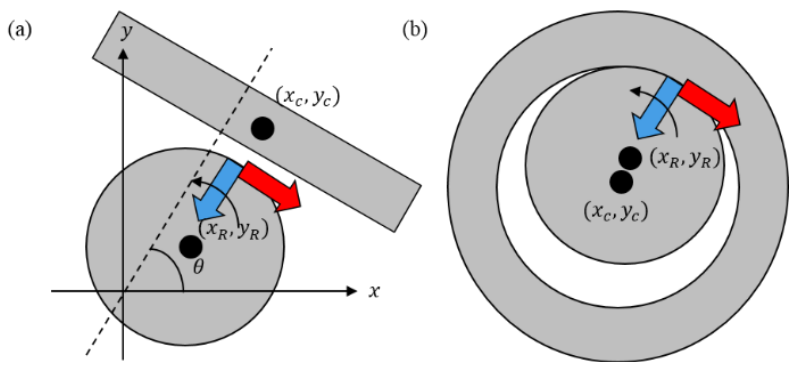

Figure 1. Type of Rubbing. (a) One side contact case. (b) Annular contact case.

components in the one side contact case and the annular contact case are created for analyzing the several contact configurations respectively.

In the analysis of rubbing, models in which the rotor come into contact with the casing due to unbalance or models in which rotor is already contacting with the casing at rest are usually analyzed. However, in this research, the model in which the rotor come into contact from a non-contact state by an external force due to earth quake or flow-induced vibration is analyzed. The relationship between the contact configurations and the generation of various kinds of vibration is investigated. We validated the rubbing model in one side contact case with a rotor kit. By simulation, we reproduced the time history, the orbit and the full spectrum characteristics of the rotating shaft measured by the experiment precisely.

\section{Rubbing Forces and Equations}

This section describes the modeling of the rubbing force between the rotor and the casing. In Figure 1, schematic overviews of the rotor and the casing in rubbing are shown. The two contact configurations, one side and annular contact cases for translational motion are treated. The rubbing force consists of the radial contact force (blue arrow in Figure 1) and the tangential friction force (red arrow in Figure 1).

Contact stress theory is used for the contact force model. The contact force between the rotor and the casing is modeled as a piecewise linear spring and damper model. The friction force is modeled by multiplying the contact spring force by the friction coefficient. Although there is a model for using the coefficient of restitution for contact, since the contact time becomes infinitely small, it is not suitable for handling the frictional force which is calculated by multiplying the contact spring force by the friction coefficient at the time of contact.

The contact spring force $F_{\mathrm{s}}$ and the damping force $F_{\mathrm{d}}$ and the friction force $F_{\mathrm{f}}$ are written as follows.

$$
\begin{aligned}
& F_{\mathrm{S}}(r)= \begin{cases}K\left(r-C_{R}\right) & \text { if } r>C_{R} \\
0 & \text { if } r \leq C_{R}\end{cases} \\
& F_{\mathrm{d}}\left(v_{r}\right)=\left\{\begin{array}{cc}
d v_{r} & \text { if } r>C_{R} \\
0 & \text { if } r \leq C_{R}
\end{array}\right. \\
& F_{\mathrm{f}}=\mu\left(v_{\phi}\right) F_{\mathrm{s}}(r)
\end{aligned}
$$

Here,

$C_{R}$ : Clearance,

$K$ : Contact spring constant,

$d$ : Contact damping constant,

$\mu$ : Friction coefficient.

Thus the rubbing force in one side contact at the angle $\theta$ of the rectangular components $F x$ and $F y$ are written as follows.

$$
\begin{aligned}
& F_{x}=\left(F_{\mathrm{s}}(r)+F_{d}\left(v_{r}\right)\right) \cos \theta-\mu F_{\mathrm{s}}(r) \sin \theta \\
& F_{y}=\left(F_{\mathrm{s}}(r)+F_{d}\left(v_{r}\right)\right) \sin \theta+\mu F_{\mathrm{s}}(r) \cos \theta
\end{aligned}
$$

Here, $(r, \phi)$ is the relative rotor position against the casing in the polar coordinates and $\left(v_{r}, v_{\phi}\right)$ is the relative velocity.

$$
\begin{gathered}
r=\sqrt{\left(x_{R}-x_{C}+\delta_{x}\right)^{2}+\left(y_{R}-y_{C}+\delta_{y}\right)^{2}} \cos (\phi-\theta) \\
\phi=\tan ^{-1} \frac{y_{R}-y_{C}}{x_{R}-x_{C}} \\
v_{r}=\dot{r} \\
v_{\phi}=-\left(\dot{x_{R}}-\dot{x_{C}}\right) \sin \theta+\left(\dot{y}_{R}-\dot{y}_{C}\right) \cos \theta+R \omega
\end{gathered}
$$

Here,

$\left(\delta_{x}, \delta_{y}\right)$ : The rotor offset against the casing,

$\left(x_{R}, y_{R}\right)$ : Center of the rotor,

$\left(x_{C}, y_{C}\right)$ : Center of the casing,

$R$ : Rotor radius,

$\omega$ : Rotating speed.

In the annular contact case, $\phi=\theta$ holds in Equation 4, 5 and 8.

To estimate the contact spring constant, the Hertzian Contact Theory between two cylinders with parallel axes is used (Inagaki et al, 2005). The indentation depth $r-C_{R}$ is related to the contact force $F_{\mathrm{c}}$ as follows.

$$
\begin{gathered}
\left(r-C_{R}\right)=\frac{2 F_{\mathrm{c}}\left(k_{0}+k_{1}\right)}{\pi L}\left(1.8864+\log \left(\frac{L}{2 b}\right)\right) \\
k_{i}=\frac{1-v_{i}^{2}}{E_{i}}(i=0,1) \\
b=\sqrt{\frac{2 F\left(k_{0}+k_{1}\right) R}{\pi l}} \\
\frac{1}{R}=\frac{1}{R_{0}}+\frac{1}{R_{1}}
\end{gathered}
$$

Here,

$v_{i}$ : Poisson ratio of the cylinder,

$E_{i}$ : Young's modulus of the cylinder,

$R_{i}$ : Radius of the cylinder,

$L$ : Length of the cylinder.

The contact spring constant is estimated by linearizing the contact force $F_{\mathrm{c}}$ against the indentation depth $r-C_{R}$.

The contact damping constant is estimated so that the loss for one contact is equivalent to that calculated from the coefficient of restitution. The coefficient of restitution $e$ is defined by the following equation,

$$
e=-\frac{v_{1}}{v_{0}}
$$


Here, $v_{1}$ is the vertical velocity of the rotor immediately after contact with the casing surface, $v_{0}$ is the velocity immediately before contact.

Assuming that the rotor motion in contact follows the damped harmonic oscillator, the following relationship holds.

$$
\begin{gathered}
v_{1}=v_{0} \exp \left(-\frac{\pi \zeta}{\sqrt{1-\zeta^{2}}}\right) \\
\zeta=\frac{d}{2 \pi \sqrt{m K}}
\end{gathered}
$$

Here,

$\zeta$ : Damping ratio, $m$ : Rotor mass.

From Equation 13, 14 and 15, the contact damping constant $d$ is given by the function of the coefficient of restitution $e$ as follows

$$
d=\sqrt{\frac{2(\log e)^{2} m K}{\pi^{2}+(\log e)^{2}}}
$$

It is possible to determine the coefficient of restitution by an experiment or the other more detailed analysis (Jackson et al, 2009).

\section{Modelica Implementation}

The presented rubbing force models must be supplied by constraints in the transverse direction $x, y$ and rotating angle direction. Our Rotating Machinery library is used to supply these constraints (Ishibashi et al, 2017). The presented rubbing models are implemented in our rotating machinery library. The basic flange of this library has 5 DOF (degree of freedom), consisting of 4 DOF (two dimensional deflections and slopes) for transverse vibration of the rotor system and 1 DOF (rotating angle) for torsional vibration, neglecting axial vibration. Features like unbalanced rotors, flexible beams (shaft), supports, springs and dampers are all represented. The library is used to create the total rotating machinery system.

The rubbing force components in the one side contact case and the annular contact case are implemented respectively. The rubbing force components are implemented with two connectors, each with 5 DOF. Since the above rubbing force models has the only 3 DOF, the moments are set as zero. These connectors are the connections to the rotor and the casing.

The contact spring and damping force are implemented by the same method as that of Modelica. Mechanics. Translational. Componen ts.ElastoGap. In order to calculate the friction force without further discontinuous events, the continuously differentiable friction model which decreases the simulation speed (Makkar et al, 2005) is used. The implementation is done by the same method as the
Idealized Contact library (Oestersötebier et al, 2014). The following function of the relative velocity $v_{\phi}$ to approximate the friction coefficient of the characteristic Stribeck curve is implemented.

$$
\begin{array}{r}
\mu\left(v_{\phi}\right)=\gamma_{1}\left(\tanh \left(\gamma_{2} v_{\phi}\right)-\tanh \left(\gamma_{3} v_{\phi}\right)\right) \\
+\gamma_{4} \tanh \left(\gamma_{5} v_{\phi}\right)+\gamma_{6} v_{\phi}
\end{array}
$$

Here,

$\gamma_{i}(i=1,2, \cdots, 6)$ : Non-physical constants.

In Figure 2 the icons of the rubbing force components in the one side contact case and the annular contact case are shown. No inertias or constraints are included in the model.

Using our Rotating Machinery library, it is possible to create rotating machinery systems. A simple rotating machinery system with casing is easily generated. Here, we treat a Jeffcott rotor system in partial rubbing with the casing as a test case in Figure 3. The model parameters are set to simulate the rotor kit shown in Figure 14. The lowest eigen frequency $\varepsilon_{L}$ of the shaft bending mode in the model is $30 \mathrm{~Hz}$. The casing mass, stiffness and damping are the same as the rub screw in Figure 14. Also, the contact spring and damping constant and friction coefficient in the rubbing component are the same as the rub screw shown in Figure 14. Only the casing position of the model in the direction of rotating shaft axis is different from the model shown in Figure 18.

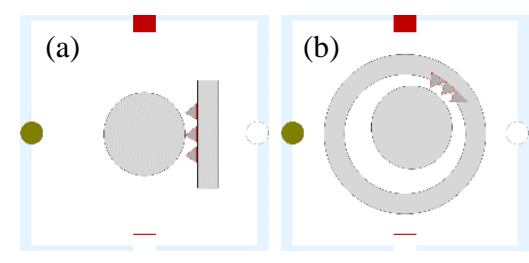

Figure 2. Modelica Icons for rubbing components. (a) One side contact case. (b) Annular contact case.

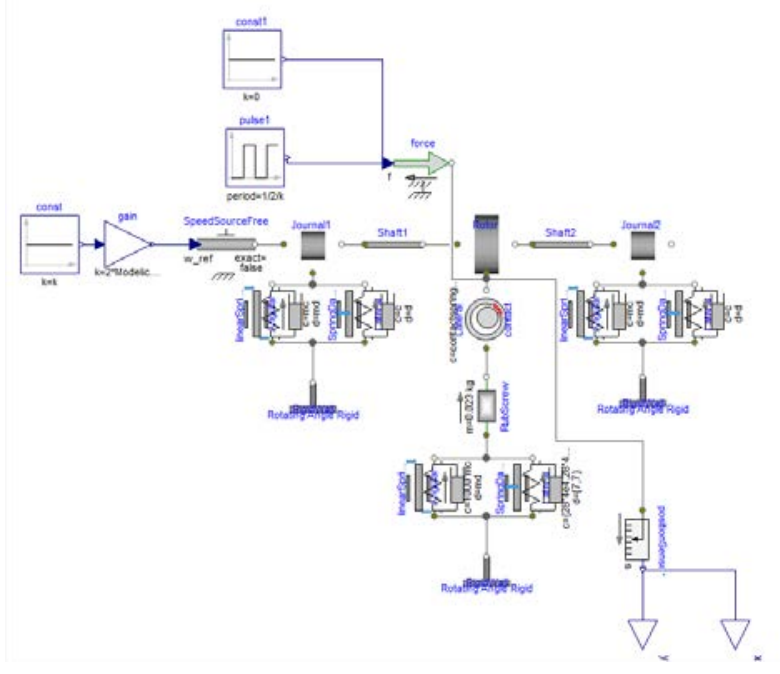

Figure 3. Modelica model of a Jeffcott rotor system having partial rubbing with the casing. 
In this model (Figure 3), the whole rotor system including the casing position is symmetrical. In the model, the rubbing component (Figure 2) is defined as described in this paper, all other components are from our rotating machinery library.

\section{Simulation Results}

In this section, using the model (Figure 3) and rubbing components (Figure 2), the generation of various kinds of vibration at high speed rotating speed due to partial rubbing is investigated by simulations.

\subsection{Rubbing vibration}

Dymola is used for the simulations. Since the model contains many events, single-step solver "Radau" is used for simulation.

Simulation is done at the constant rotating speed $\omega$ over 1.5 times the speed of the critical speed $\varepsilon_{L}$. The simulation that the rotating shaft is whirling with smaller amplitude than the clearance is done. By applying a pulsating external force, the partial rubbing vibration is induced (Figure 4). A pulsating external force is applied when the gap between the rotor and the casing become the smallest.

As a result, although the rotor whirling of unbalance is smaller than the clearance, the rotor keeps in contact with the case after the contact due to a pulsating external force under some conditions despite the same pulsating external force amplitude (Figure 4). However, the vibration converges and returns to a non-contact state under another conditions (Figure 5). To investigate this kind of vibration, batch simulation of sweeping rotating speed and the eccentricity of static unbalance in Rotor is done by python interface.

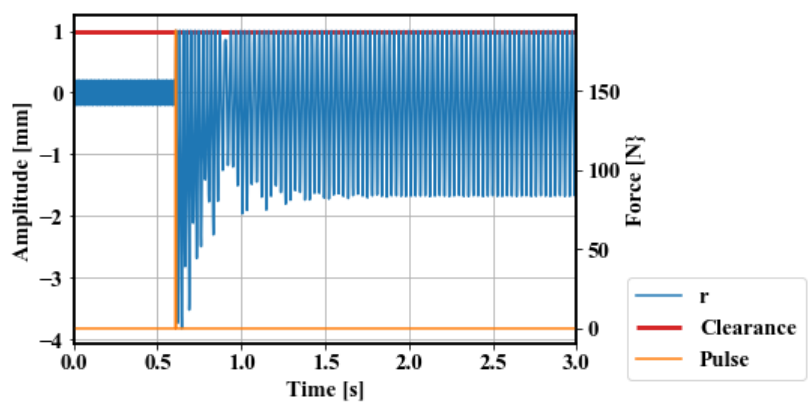

Figure 4. Rubbing vibration occurs and continues.

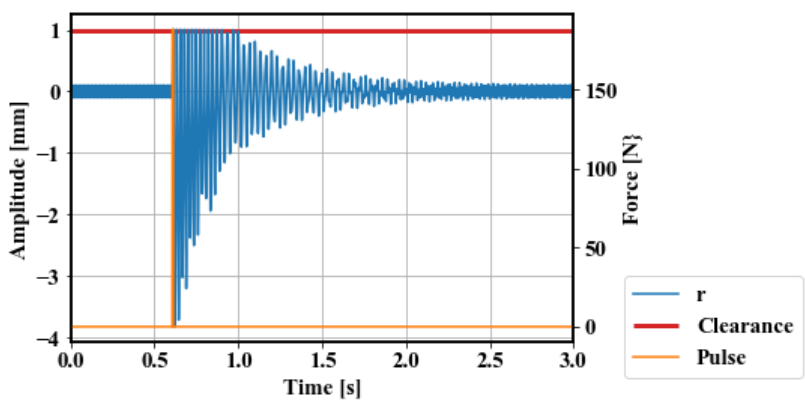

Figure 5. Rubbing vibration does not occur.

\subsection{One side contact case}

Figure 6 shows the domain of the rubbing vibration occurrence in the one side contact case. The model (Figure 3) replacing the rubbing component in the annular contact case with that in the one side contact case is simulated. The contact angle $\theta=\pi / 2$ and the clearance $C_{R}=1 \mathrm{~mm}$ are set in the rubbing component. The rubbing (i.e. contact) vibration occurs and continues in the region with a plot in Figure 6. The vibration converges immediately after the contact, and returns to a non-contact state in the region without a plot in Figure 6 . Figure 7 shows the rubbing vibration behavior in the one side contact case. From the left figure, the Rotor displacement in Y direction, orbit and full spectrum are shown. The full spectrum is obtained from the half spectrums of each $\mathrm{X}$ and $\mathrm{Y}$ displacement by the procedure written in the paper (Goldman and Muszynska, 1999). The unbalance amplitude before contact in Figure 7 is set as around 0.1 against the clearance. Due to the translation mode of the rotating shaft, Rotor unbalance amplitude is larger in the low rotating speed range.

In the high rotating speed range over the first critical speed $\varepsilon_{L}$, the $1 / \mathrm{n}$ (n: integer) sub harmonic rubbing vibration continues in the region shown in Figure 6. These vibration occurs in higher speed of $n$ integer multiple of the eigen frequency $\varepsilon_{L}$. As the unbalance increases, the $1 / \mathrm{n}$ sub harmonic vibration occurs in higher rotating speed. As the integer $\mathrm{n}$ becomes larger, the $1 / n$ sub harmonic rubbing vibration occurs from smaller unbalance region. The domain of the rubbing vibration occurrence shows a gap. In the gap where rubbing vibration is unlikely to occur, the casing is more than clearance away and moving away from the rotor.

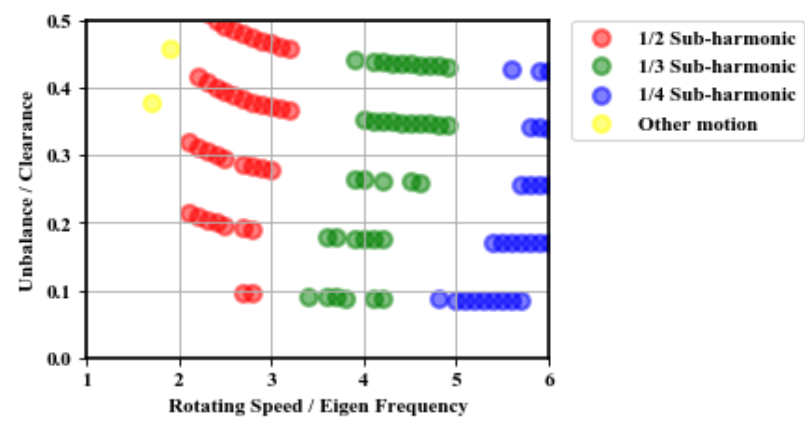

Figure 6. The domain of the rubbing vibration occurrence in the one side contact case. 

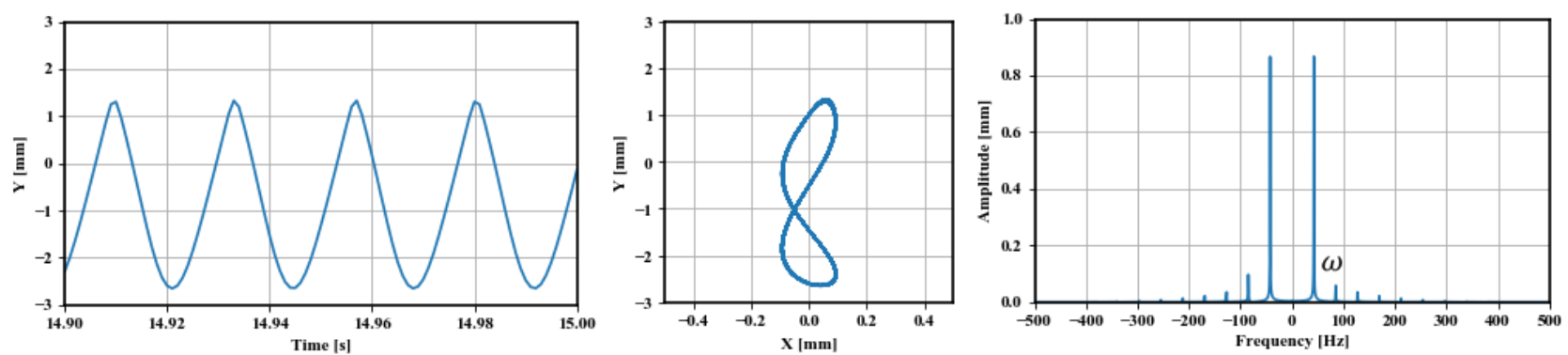

(a) $1 / 2$ Sub-harmonic Vibration $\omega / \varepsilon_{L}=2.8$
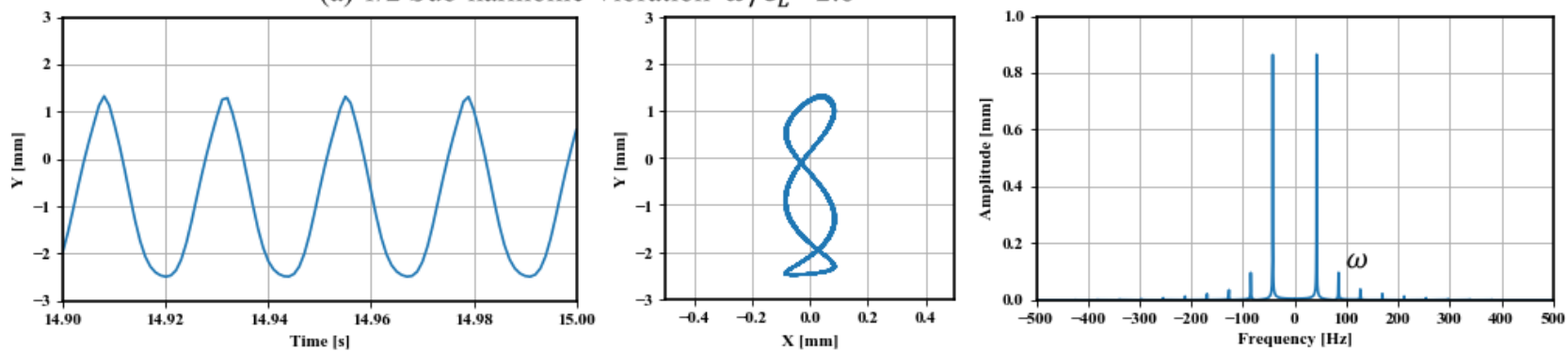

(b) $1 / 3$ Sub-harmonic Vibration $\omega / \varepsilon_{L}=4.2$
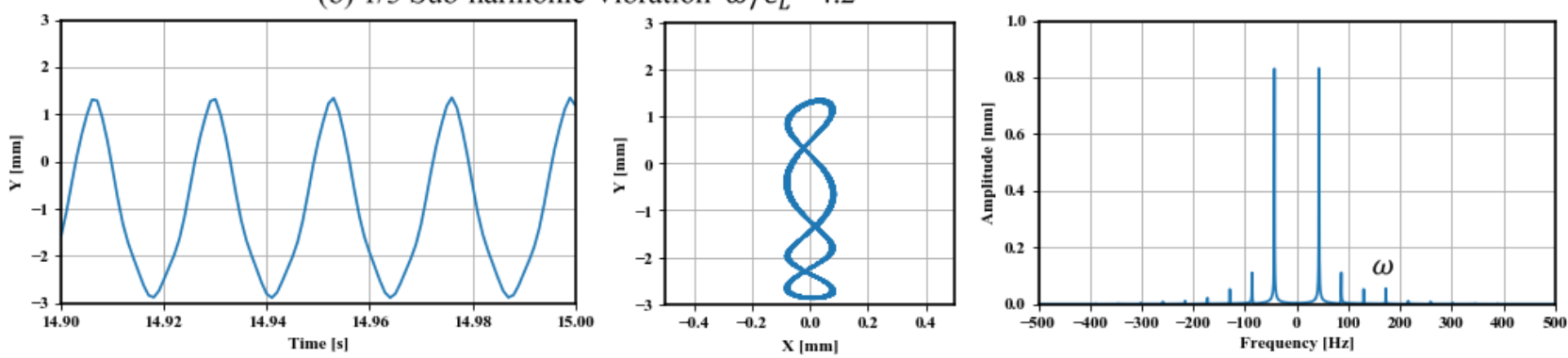

(c) $1 / 4$ Sub-harmonic Vibration $\omega / \varepsilon_{I_{L}}=5.7$
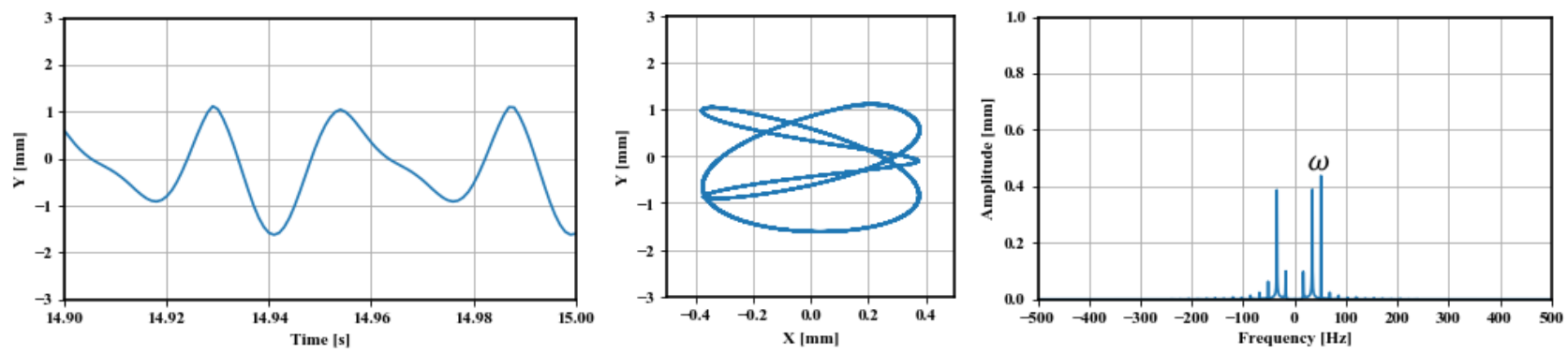

(d) Other motion $\omega / \varepsilon_{L}=1.7$, Unbalance/Clearance $\approx 0.4$

Figure 7. Vibration behavior in the one side contact case. 


\subsection{Both side contact case}

Figure 9 shows the domain of the rubbing vibration occurrence in the both side contact case. Figure 8 shows the rubbing vibration behavior. The model adding the one side rubbing component and the casing on the other side is simulated. The two casings have the same mass, spring and damping constant as each other. Also, the two one side rubbing components have the same parameters values such as the contact spring and damping constant and friction coefficient as each other. The contact angle $\theta=-\pi / 2$ and the clearance $C_{R}=$ $1 \mathrm{~mm}$ are set in the other rubbing component.

In this case, the synchronous with rotating speed and $1 / \mathrm{n}$ sub harmonic vibration occurs. The sub harmonic rubbing vibration just occurs in the region of moderately small unbalance. As the unbalance gets larger, synchronous vibration occurs in the region of wide rotating speed range. This violent vibration is regarded as a kind of collision type self-excited vibration. In the both side contact case, only the odd number sub harmonic vibration occurs. Since the rotor orbit of the even number sub harmonic vibration is basically asymmetrical with respect to the origin, there is no solution where there are two casings located on both sides of the rotor under the same condition (see Figure 7 and Figure 8). However, if there is a difference between the two casings, the rotor sometimes contacts with the casings only at one side.

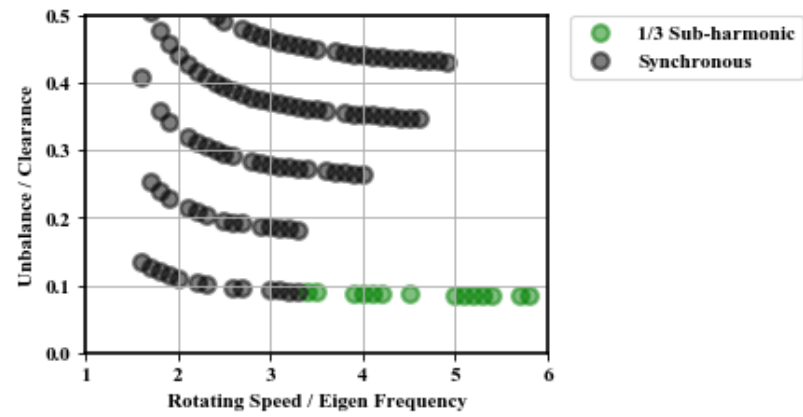

Figure 9. The domain of the rubbing vibration occurrence in the both side contact case.
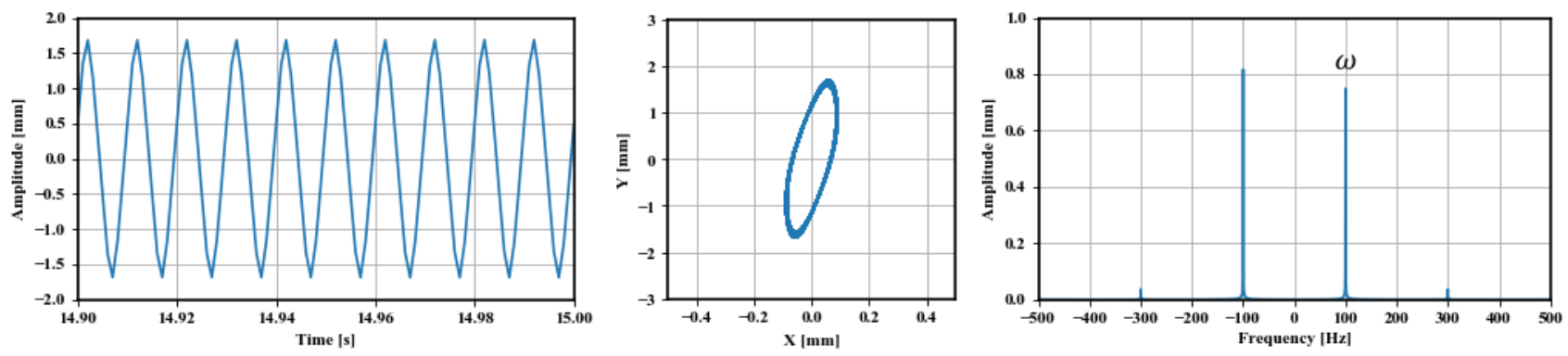

(a) Synchronous Vibration $\omega / \varepsilon_{L}=3.3$
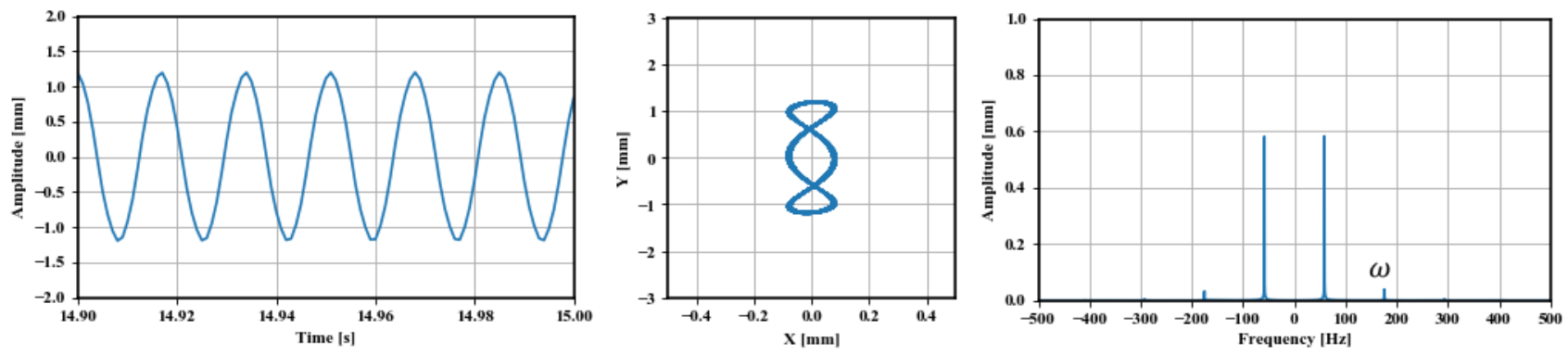

(b) 1/3 Sub-harmonic Vibration

$\omega / \varepsilon_{L}=5.8$

Figure 8. Vibration behavior in the both side contact case. 


\subsection{Annular contact case}

Figure 11 shows the domain of the rubbing vibration occurrence in the annular contact without offset. The model (Figure 3) is simulated. Figure 10 shows the rubbing vibration behavior. The contact spring constant in the rubbing component is calculated by considering the difference in the curvature. In this case, two kinds of collision type self-excited synchronous vibration with rotating speed occurs. One is the circle rotor orbit and the other is the oval rotor orbit shown in Figure 10. In the low rotating speed range, synchronous circle rotor orbit vibration occurs. In the high rotating speed range, synchronous oval rotor orbit vibration occurs from the small unbalance region. From the rotor orbit in Figure 10 , the rotor collides with the casing several times per one whirling period.

Figure 12 shows the domain of contact vibration in the annular contact case with offset. Figure 13 shows the rubbing vibration behavior. The model (Figure 3 ) with the rotor offset $\left(\delta_{x}, \delta_{y}\right)=\left(0, C_{R} / 4\right)$ against the casing in the rubbing component is simulated. In comparison with the domain without the offset, the synchronous circle rotor orbit vibration occurs in the wider rotating speed range toward high rotating speed. In addition to the synchronous vibration in the annular contact case without the offset, the sub harmonic and the other kind of the synchronous circle rotor orbit vibration occur. The sub harmonic vibration occurs in the slightly higher rotating speed of the $\mathrm{n}$ integer multiple of the eigen frequency $\varepsilon_{L}$.

Although there was a small difference between our simulation results and the previous papers (Watanabe et al, 2004; Watanabe et al, 2005) due to the differences of the parameters amplitudes in the rubbing component, the relationship between the contact configurations and the generation of various kinds of vibration obtained in this paper showed the same trend as those papers.

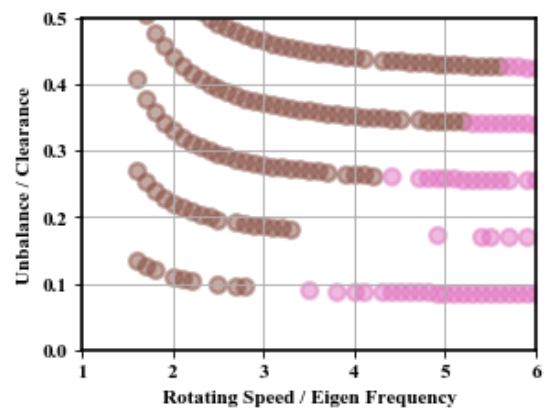

Synchronous Circle Synchronous Oval

Figure 11. The domain of the rubbing vibration occurrence in the annular contact case without the offset.
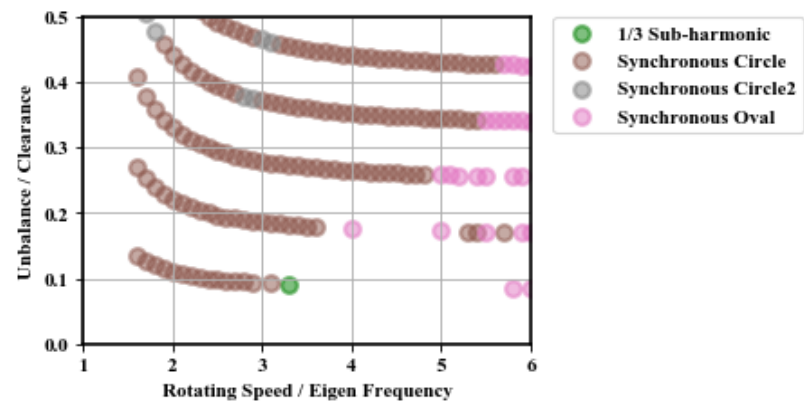

Figure 12. The domain of the rubbing vibration occurrence in the annular contact case with the offset.
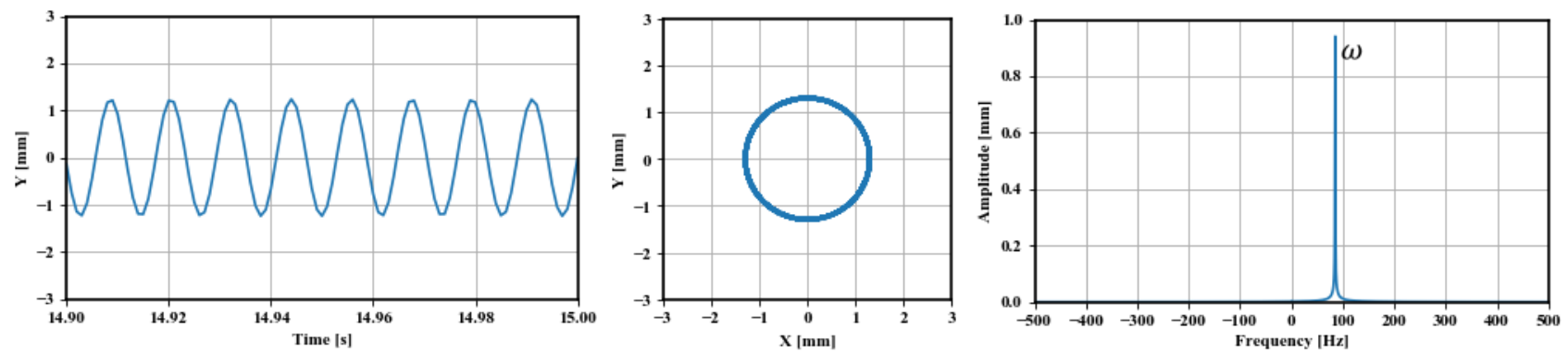

(a) Collision type Synchronous Vibration (Circle Orbit) $\omega / \varepsilon_{L}=2.8$
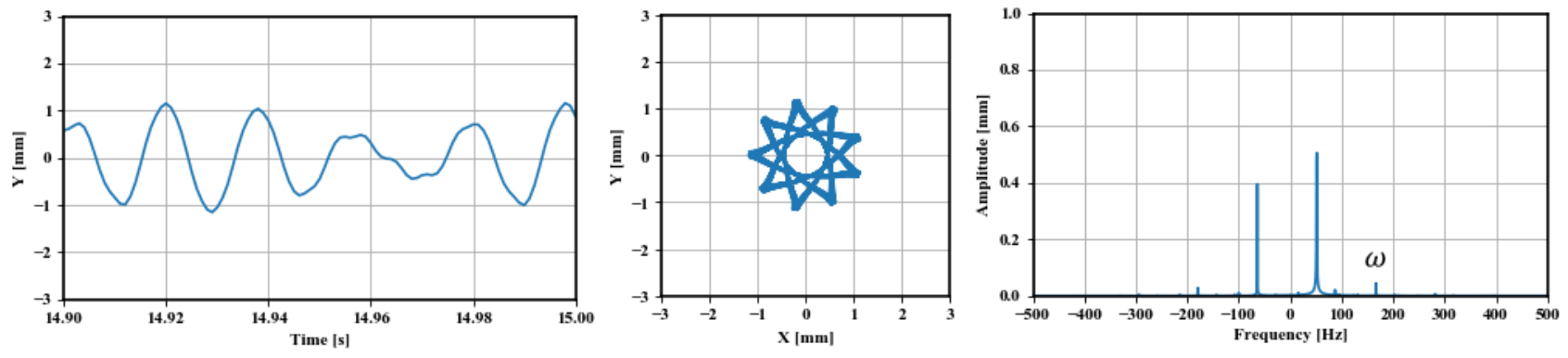

(b) Collision type Synchronous Vibration (Oval Orbit) $\omega / \varepsilon_{L}=5.5$

Figure 10. Vibration behavior in the annular contact case without the offset. 

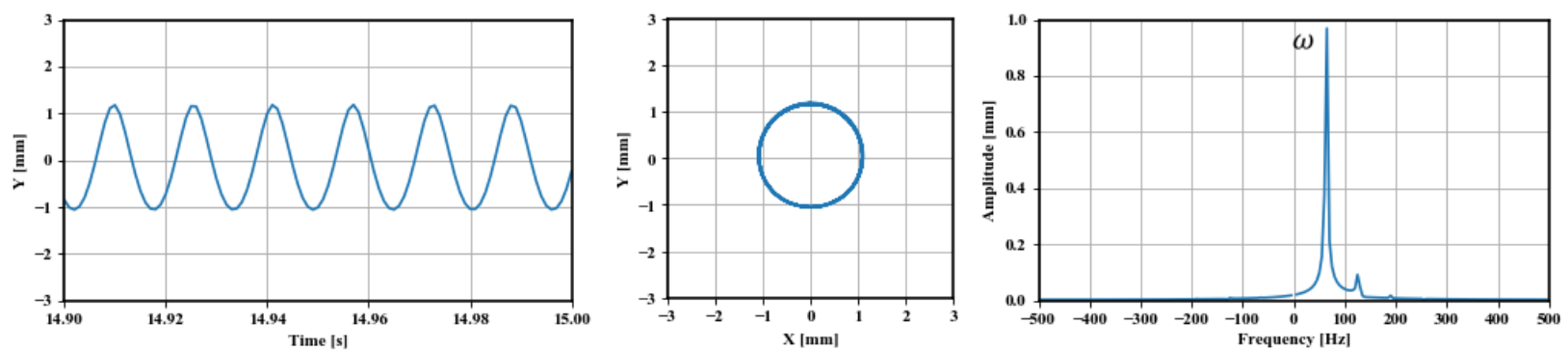

(a) Collision type Synchronous Vibration (Circle Orbit) $\omega / \varepsilon_{L}=2.1$
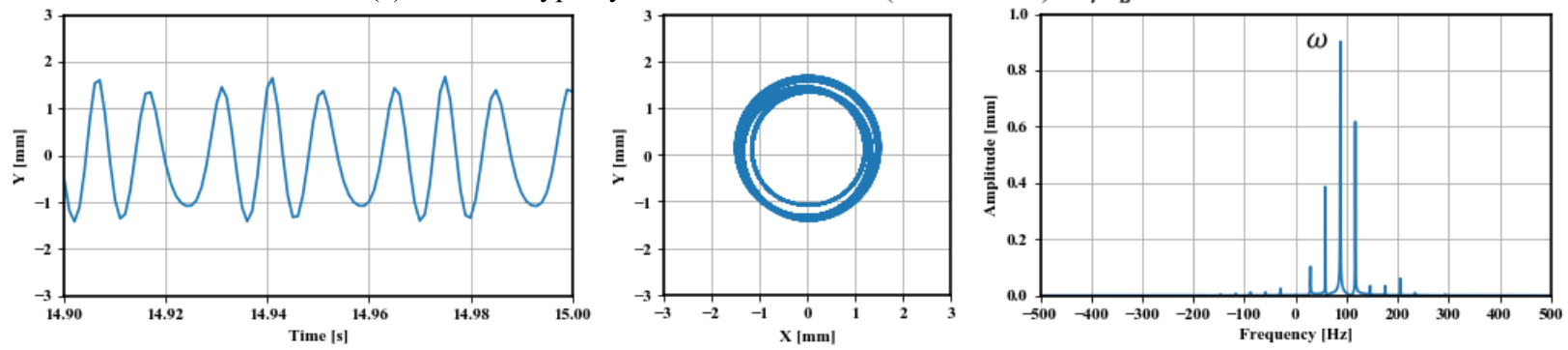

(b) Collision type Synchronous Vibration (Circle Orbit 2) $\omega / \varepsilon_{L}=2.9$, Unbalance/Clearance $\approx 0.4$
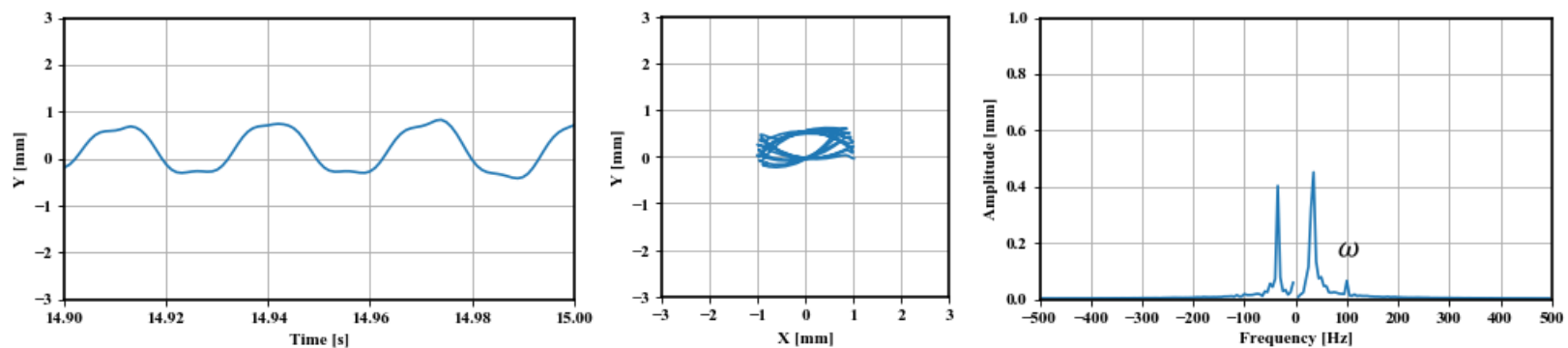

(c) $1 / 3$ Sub-harmonic Vibration $\omega / \varepsilon_{L}=3.3$
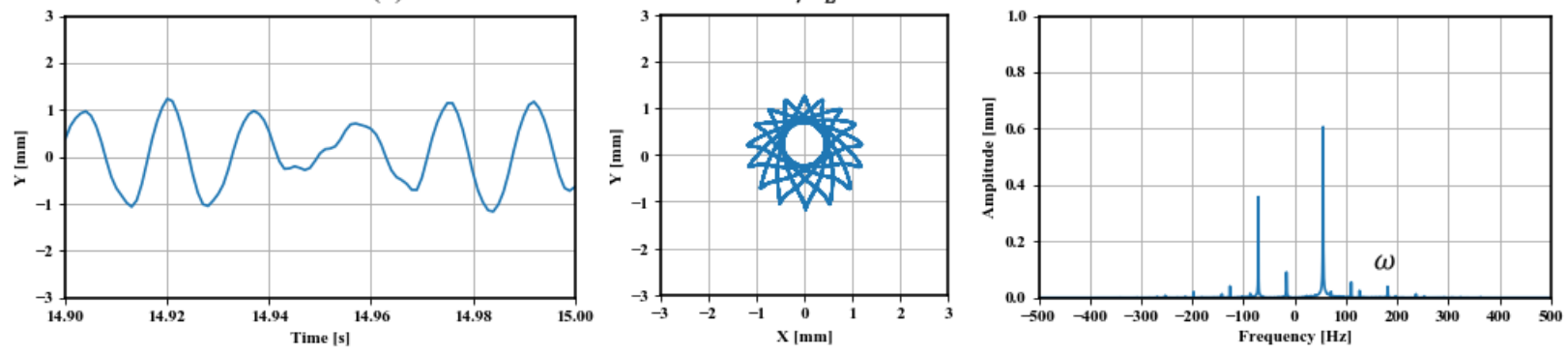

(d) Collision type Synchronous Vibration (Oval Orbit) $\omega / \varepsilon_{L}=6.0$

Figure 13. Vibration behavior in the annular contact case with the offset.

\section{Model Validation}

\subsection{Experiment}

To validate our models, we used the rotor kit (RK 4 Rotor Kit GE Bently Nevada) shown in Figure 14. The rotating shaft was supported by the solid lubricated bearings covered by the rubber. The rotating shaft was measured by two proximitors arranged in orthogonal directions. The data sampling time of the proximitors for recording was $1 \mathrm{~ms}$.

To make it easier for analyzing the experimental result, the Jeffcott rotor system was investigated for the experiment. One side contact condition was established by adjusting the rub screw made of brass and applying a pulsating external force to the rotating shaft.
From the preliminary impulse and rotating speed ramp response experiment, the first critical speed $\varepsilon_{L}$ (the lowest eigen frequency of the shaft bending mode) of this rotating shaft system was around 30 rps. By rotating at 70 rps (over twice the first critical speed) and applying a pulsating external force, the rubbing condition was established.

Figure 15 shows the time history of the rotating shaft in the both horizontal and vertical direction under rubbing condition. Figure 16 shows the orbit of the rotating shaft. Figure 17 shows the full spectrum analysis of the rotating shaft time history. The procedure for obtaining the full spectrum from the half spectrums of each proximity probe was followed by the paper (Goldman and Muszynska, 1999). The 1/2 sub harmonic rubbing vibration was observed. 


\subsection{Simulation}

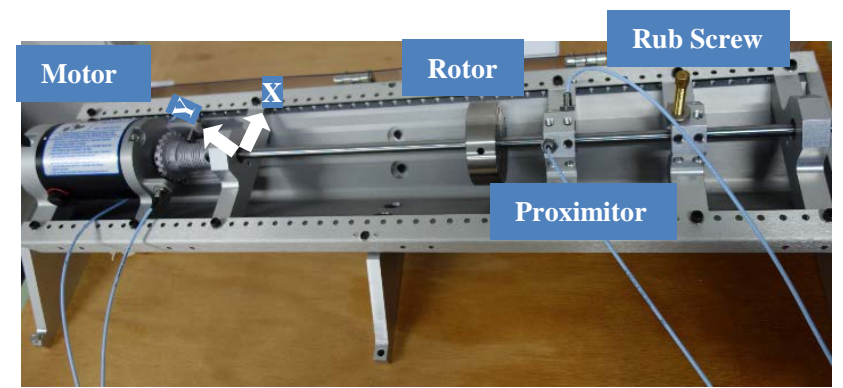

Figure 14. The rotor kit.
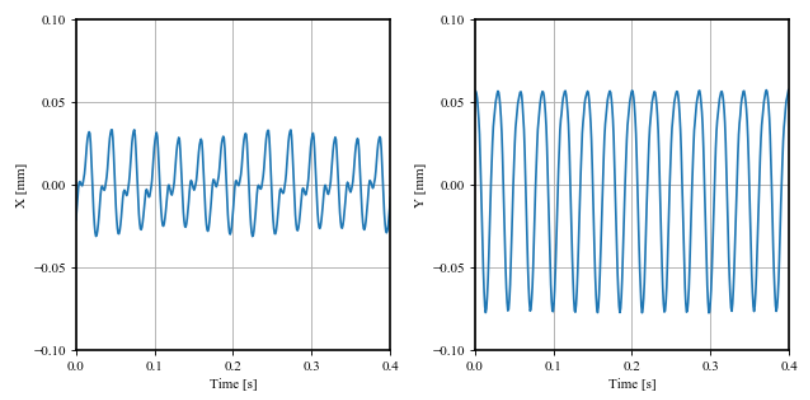

Figure 15. The time history of the experiment under rubbing condition at the rotating speed of 70 rps.

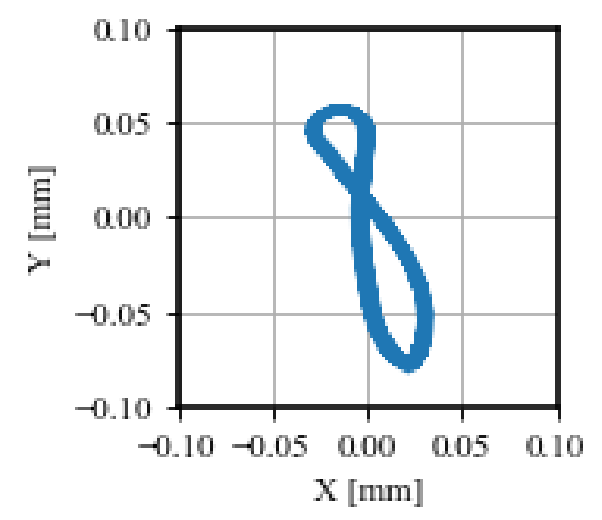

Figure 16. The orbit of the experiment under rubbing condition at the rotating speed of 70 rps.

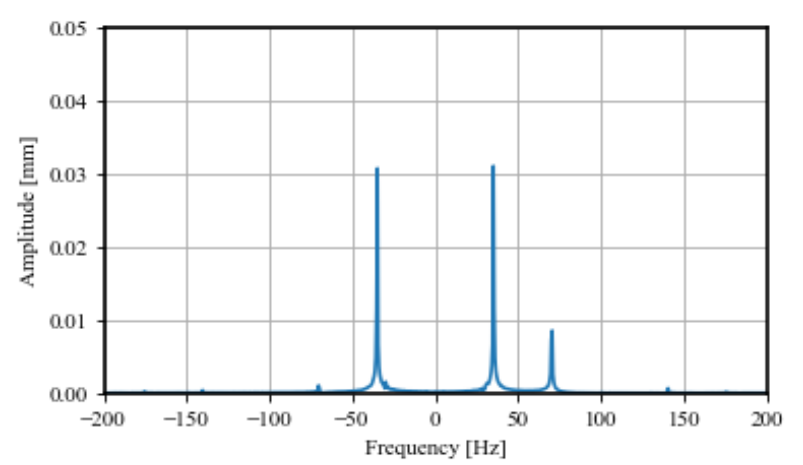

Figure 17. The full spectrum analysis of the experiment under rubbing condition at the rotating speed of 70 rps.

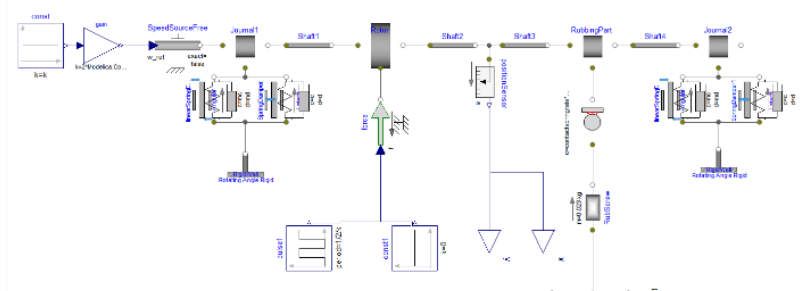

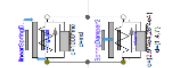

I.

Figure 18. Modelica model for the rotor kit.
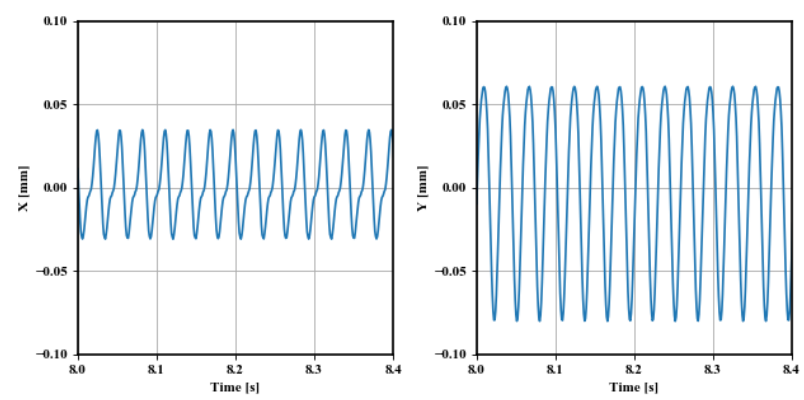

Figure 19. The time history of the simulation under rubbing condition at the rotating speed of 70 rps.

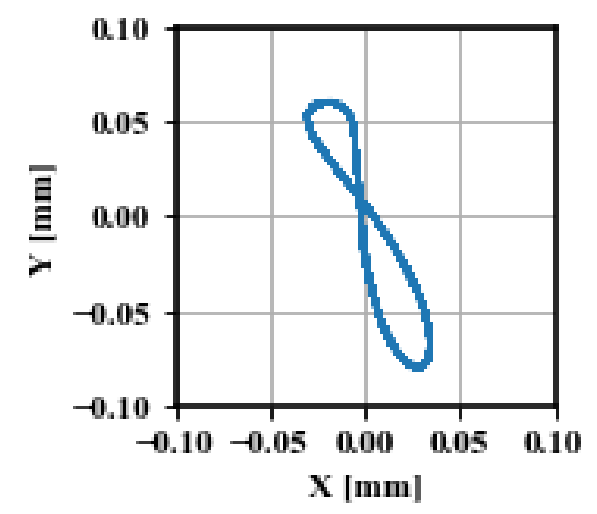

Figure 20. The orbit of the simulation under rubbing condition at the rotating speed of 70 rps.

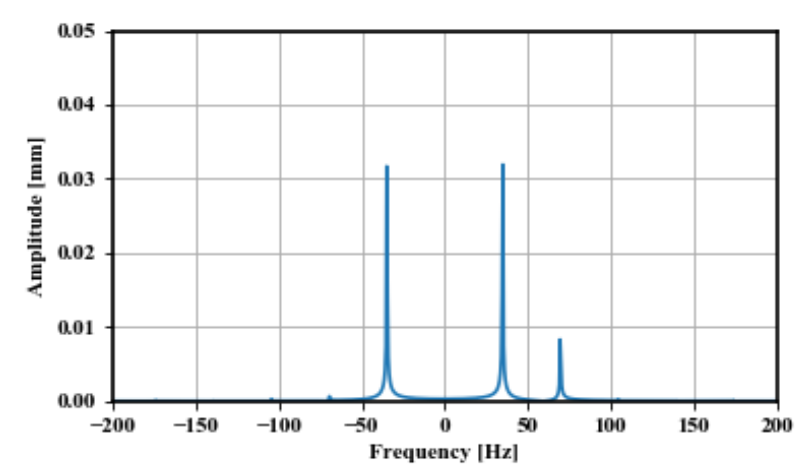

Figure 21. The full spectrum analysis of the simulation under rubbing condition at the rotating speed of 70 rps. 
To carry out the analytical investigation of the experimental result, we built the Modelica model corresponding to the rotor kit based on our library shown in Figure 18. The parameters such as the bearing stiffness and damping, the residual bow (the bend of shaft) and the rotor static unbalance of the model were calibrated to match the preliminary experiments by the same method of the previous paper (Ishibashi et al, 2017). The rub screw stiffness and damping were estimated by the preliminary impulse test. The contact spring constant in the rubbing component were estimated by Equation 9. The contact damping constant were estimated by Equation 16 using the approximate value of the coefficient of the restitution (Jackson et al, 2009). The friction coefficient was estimated from the value reported in the paper (Watanabe et al, 2004).

Figure 19 - Figure 21 show the results under rubbing condition. By simulation, we reproduced the time history, the orbit and the full spectrum characteristics of the rotating shaft measured by the experiment precisely.

\section{Conclusions}

In this paper, the rubbing component models in the one side contact case and the annular contact case are presented to describe the partial rub. Using our rotating machinery library, it is possible to model a rotating machinery system with partial rubbing.

Examples of a Jeffcott rotor system with the different contact configuration were investigated by simulation. The relationship between the contact configurations and the generation of various kinds of vibration such as "collision type synchronous vibration", "sub harmonic vibration", etc. obtained by simulation showed the same trend as the previous papers (Watanabe et al, 2004; Watanabe et al, 2005).

We validated our model with partial rubbing in the one side contact case with a rotor kit. By simulation, we reproduced the time history, the orbit and the full spectrum characteristics of the rotating shaft measured by the experiment precisely.

The presented models make it possible to carry out analytical investigations of the partial rub in order to gain some insight into the diagnostics of rotating machinery.

\section{References}

R. F. Beatty. Differentiating rotor response due to radial rubbing. Journal of Vibration and Acoustics. 107(2): 151160, 1985. doi:10.1115/1.3269238.

Yeon-Sun Choi and Sherif T. Noah. Nonlinear Steady-State Response of a Rotor-Support System. Journal of Vibration, Acoustics, Stress and Reliability in Design. 109 (4), 225261, 1987. doi:10.1115/1.3269429.

Markus Dahl, Håkan Wettergren and Henrik Tidefelt. Modelica Spur Gears with Hertzian Contact Forces. Proceedings of the 12th International Modelica Conference, 2017. doi:10.3384/ecp17132755.
Fredric F. Ehrich. Subharmonic vibrations of rotors in bearing clearance, ASME Paper 66-MS-1, 1966.

Paul Goldman and Agnes Muszynska. Application of full spectrum to rotating machinery diagnostics. Orbit First Quarter, pp. 17-21, 1999.

Andreas Hofmann, Lars Mikelsons, Ines Gubsch, and Christian Schubert. Simulating Collisions within the Modelica MultiBody Library. Proceedings of the 10th International Modelica Conference, 2014. doi: 10.3384/ecp14096949.

Mizuho Inagaki, Yukio Ishida and Akimasa Hayashi. Nonlinear Resonances and Self-Excited Oscillations of a Rotor due to Radial Clearance and Impact in Bearings. Trans. JSME, C 71, pp. 2113-2118, 2005 (in Japanese). doi: 10.1299/kikaic.71.2113.

Tatsuro Ishibashi, Han Bing and Tadao Kawai. Rotating Machinery Library for Diagnosis. Proceedings of the 12th International Modelica Conference, 2017. doi:10.3384/ecp17132381.

Robert L. Jackson, Itzhak Green and Dan B. Marghitu. Predicting the coefficient of restitution of impacting elasticperfectly plastic spheres. Nonlinear Dyn, 60: 217-229, 2010. doi: 10.1007/s11071-009-9591-z.

Ivan Kosenko and Il'ya Gusev. Implementation of the spur involute gear model on modelica. Proceedings of the 8th International Modelica Conference, 2011. URL https://www.modelica.org/events/modelica2011/Proceeding s/pages/papers/13_3_ID_117_a_fv.pdf.

Ivan Kosenko and Ilya Gusev. Revised and improved implementation of the spur involute gear dynamical model. Proceedings of the 9th International Modelica Conference, 2012. doi:10.3384/ecp12076311.

C. Makkar, W. E. Dixon, W. G. Sawyer, and G. Hu. A New Continuously Differentiable Friction Model for Control Systems Design. Proceedings of the 2005 IEEE/ASME International Conference on Advanced IntelligentMechatronics, Monterey CA, July, 2005.

Felix Oestersötebier, Peng Wang and Ansgar Trächtler. A Modelica Contact Library for Idealized Simulation of Independently Defined Contact Surfaces. Proceedings of the 10th International Modelica Conference, 2014. doi: 10.3384/ecp14096929.

Martin Otter, Hilding Elmqvist, and José Díaz López. Collision Handling for the Modelica MultiBody Library. Proceedings of the 4th International Modelica Conference, 2005. URL http://elib.dlr.de/12299/1/otter2005-modelicacollision.pdf

F.L.J. van der Linden. Modelling of elastic gearboxes using a generalized gear contact model. Proceedings of the 9th International Modelica Conference, 2012. doi:10.3384/ecp12076303.

Yusuke Watanabe, Takuzo Iwatsubo and Keizo Awa. Study of Rotor Vibration due to the Rubbing against Casing. Trans. JSME, C 70, pp. 2181-2187, 2004 (in Japanese). doi: 10.1299/kikaic.70.2181.

Yusuke Watanabe, Takuzo Iwatsubo and Keizo Awa. Study of Rotor Vibration due to the Rubbing against Casing. Trans. JSME, C 71, pp. 1421-1428, 2005 (in Japanese). doi: 10.1299/kikaic.71.1421. 